\title{
«Wir für uns» - Menschen mit geistiger Behinderung setzen sich für ihre Anliegen ein
}

\author{
Eine Gruppe von Betroffenen hat einen Flyer entworfen, der Ärzte informiert, worauf \\ sie im Umgang mit Menschen mit geistiger Behinderung besonders achten sollten.
}

\section{Felix Brem}

Präsident der SAGB

(Schweizerische Arbeitsgemeinschaft von Ärzten für Menschen mit geistiger oder mehrfacher Behinderung) und des VBMB (Verein für eine bedürfnisgerechte medizinische Versorgung für Menschen mit geistiger oder mehrfacher Behinderung)
Korrespondenz:

Dr. med. Felix Brem

Facharzt Psychiatrie und

Psychotherapie FMH

Rathausstrasse 17

CH-8570 Weinfelden

Tel. 0716269070

felix.brem[at]hin.ch
Die Arzt-Patientenbeziehung bedarf bei Menschen mit geistiger Behinderung besonderer Achtsamkeit. M. Burbidge erwähnte 1999 in einem Artikel [1], dass sich betroffene Patienten über Ärzte beklagten, die

- schreien;

- über die Betroffenen (hinweg)reden, so als ob sie gar nicht da wären;

- nicht erklären, was passiert;

- die Patientinnen und Patienten behandeln, als ob sie blöd wären;

- gar nicht zuhören, wenn der Patient etwas sagen möchte;

- so tun, als ob sie verstehen würden, obwohl sie offensichtlich gar nichts von dem verstehen, was der Patient sagt;

- den Patientinnen und Patienten nicht genug Zeit geben, um sich auszudrücken.

Im Kanton St. Gallen bildete sich in den vergangenen Jahren eine Gruppe von Menschen mit geistiger Behinderung, die unter der Bezeichnung «Wir für uns» (http://wir-für-uns.ch) einen Flyer entworfen

\section{Was Sie in der Behandlung mit mir beachten sollten!}

\section{- Hören Sie mir gut zu \\ o Lassen Sie mich ausreden \\ o $\quad$ Sprechen Sie mich mit Sie an \\ o Sagen Sie es mir, bevor Sie mich anfassen \\ - Sprechen Sie mich an, nicht meine Begleitperson \\ - Erklären Sie mir alles in einfacher Sprache \\ o Nehmen Sie sich auch ein bisschen mehr Zeit auch \\ für meine psychischen Probleme \\ o $\quad$ Klären Sie mich auch über Alternativmedizin auf \\ o Ich habe Angst vor weisser Kleidung \\ ○ Ich wünsche mir eine regelmässige Abklärung \\ o Ich habe Angst vor Spritzen}

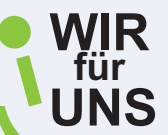

Das wünschen sich Behinderte von ihrer Ärztin oder ihrem Arzt.
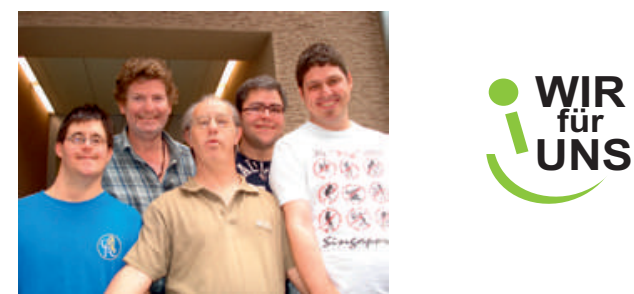

Geben Sie uns eine Chance!

hat. Für betroffene Menschen hat sie eine kleine Karte mit Hinweisen zusammengestellt, worauf der Arzt bei der Behandlung besonders achten sollte (siehe Abbildung unten) [2].

Die Schweizerische Arbeitsgemeinschaft von Ärzten für Menschen mit geistiger oder mehrfacher Behinderung SAGB (www.sagb.ch), die sich in ihren Zielsetzungen gerade für die Anliegen dieser Patientengruppe einsetzen möchte, hat dieses Anliegen aufgenommen und gemeinsam mit zahlreichen anderen Organisationen, auf ärztlicher Seite speziell unterstützt von FMH, FMPP, SGIM, SGEP, SGP eine Tagungsserie vorbereitet unter dem Titel «Bedürfnisgerechte Medizin für alle», die am 1. September 2012 mit einer Impulstagung starten wird (www. vbmb.ch).

Es scheint uns wichtig, diese Anliegen als kleinen Beitrag zur Qualitätsverbesserung der ganzen Ärzteschaft bekannt zu machen; bei Menschen mit geistiger Behinderung gilt nämlich noch mehr als ohnehin:

«Schwierigkeiten in der Kommunikation stellen eine der wichtigsten Hürden auf dem Weg zu einer qualitativ hochwertigen medizinischen Versorgung dieser Patientengruppe dar» (M. Burbidge) [1].

\footnotetext{
Referenzen

1 Burbidge M. In: Lennox N, Diggens J (Hrsg.). Management Guidelines - People with Developmental and Intellectual Disabilities; 1999.

2 Die Flyer sind im Internet zugänglich unter der Adresse: http://wir-für-uns.ch/Ueber-uns/ Aerzte-Flyer/index.php
} 